\title{
Aprendizaje Basado en Problemas mediante un modelo de Teleenseñanza
}

\section{Problem-Based Learning through a Distance Learning Model}

\author{
Miriam Martínez García, David Romero Faz \\ Departamento de Ingeniería civil: Construcción, Infraestructura y Transporte, Universidad Politécnica de Madrid, España. \{miriam.martinez
} david.romero\}@upm.es

\begin{abstract}
Resumen
Durante los cursos académicos 2009-10, 2010-11, 2011-12, 2012-13 y 2013-14 se han aplicado técnicas de Aprendizaje Basado en Problemas junto con la utilización de un Modelo de Sistema de Teleenseñanza (e-learning) y un con un modelo de acción tutorial, con grupos de alumnos voluntarios de los últimos cursos de la Escuela Técnica Superior de Ingeniería Civil de la Universidad Politécnica de Madrid, logrando incrementar el tiempo de dedicación e implicación con las asignaturas.

La experiencia se ha desarrollado con alumnos voluntarios matriculados en las asignaturas de Caminos (Grado), Caminos I (Antigua Ing. Téc.) y Caminos II (Antigua Ing. Téc.), ya que estas son asignaturas que históricamente han obtenido tasas de aprobados y eficiencia muy bajos, del entorno del $25 \%$.

El trabajo realizado ha consistido, en un primer lugar, en conocer las expectativas que los alumnos tienen sobre la acción tutorial, tanto al comienzo del curso como una vez finalizado este, y posteriormente trabajar con alumnos voluntarios aplicando el Aprendizaje Basado en Problemas (ABP) para la adquisición de conocimientos apoyándose en un sistema de acción tutorial basado en la tele-enseñanza.

La utilización de estas técnicas de aprendizaje ha hecho que los resultados obtenidos a lo largo de la investigación sean mucho más satisfactorias que las obtenidas sin la aplicación de estas técnicas, ya que ha dado lugar a un número de aprobados cercano al 100\%, con mejores calificaciones, destacando la reducción que se ha producido en las tasas de abandono en las asignaturas estudiadas, Ilegando a obtener niveles despreciables.
\end{abstract}

\section{Palabras Clave}

Aprendizaje Basado en Problemas; Método del Caso; Teleenseñanza; Acción Turorial; Pequeños Grupos.

\begin{abstract}
During the academic courses 2009-10, 2010-11, 2011-12, 2012-13 and 2013-14 Problem-Based Learning techniques have been applied together with an E-learning Model together with tutorial action with groups of volunteer students of the last courses of the School of Civil Engineering at the Technical University of Madrid, managing to increase the time of dedication and implication with the subjects.

The experience has been developed with volunteer students enrolled in the subjects of Road (Grade), Roads I (old curriculum) and Roads Il (old curriculum), since these are subjects that have historically obtained very low pass rates and efficiency of the environment 25\%.

The realized work has consisted, in the first place, of knowing the expectations that the students have on the tutorial, both at the beginning of the course as when it is finished, and later working with voluntary students applying Learning Based on Problems (LBP) for the knowledge acquisition resting on an tutorial system based on the e-learning.

The use of these learning techniques have made the results obtained during the investigation much more satisfactory than those obtained without the application of these techniques as it has led to a number of nearby approved 100\% better qualifications, highlighting the reduction that has occurred in dropout rates in the subjects studied, obtaining negligible levels.
\end{abstract}

\section{Keywords}

Problem-Based Learning; Case Method; e-learning; Distance Learning Model; Tutorial Action; Little Groups. 


\section{Introducción}

A través de la presente investigación se trata de aportar una visión real de la aplicación efectiva de un modelo de ABP acompañado de una fuerte Acción Tutorial junto con el uso de la TIC en la Universidad. Se hace un análisis exhaustivo de las opiniones y actitudes que muestran los estudiantes en lo que se refiere a la aplicación de esta metodología didáctica.

Se considera asimismo de gran interés para la mejora del aprendizaje conocer cuál es la opinión real de los estudiantes universitarios, ya que son los principales implicados en relación a un modelo formativo apoyado en el ABP acompañado de la Acción Tutorial y de las TIC.

En el EEES, el alumno junto con el profesorado son los ejes fundamentales que dan sentido al proceso de enseñanza-aprendizaje. Siendo el alumno el que deberá tener mayor peso específico en el proceso, ya que la actividad, entendida como el conjunto de acciones y procedimientos, ha de ser primordial en dicho proceso.

En este sentido, todos los elementos que componen el sistema deben ponerse a disposición de los alumnos para que puedan gestionar su proceso de formación.

Según Jesús Salinas Ibañez (2015) la incorporación de las TIC en el ámbito educativo configura y supone que los alumnos ya no solo son alumnos, sino que también son usuarios de la formación; ante la existencia de estos nuevos escenarios, presentes tanto en la enseñanza como en el aprendizaje, los alumnos son más activos. Característica que les hará ser más y mejor preparados para la sociedad actual en la que el cambio es parte de nuestras vidas.

Los enfoques tradicionales en la enseñanza, con una fuerte dependencia en los libros de texto, instrucción masiva, conferencias y pruebas de respuesta múltiple, están obsoletas en la era de la información (Cuban, 2001).

La mayoría de los estudios tratan de recoger y analizar perspectivas, opiniones y actitudes del profesorado hacia el uso e integración de nuevas metodologías y tecnologías en las aulas, por lo que siempre se trata de hacer un diagnóstico de la visión que tienen los docentes. Según Area (2015), esta es una de las líneas de investigación que se suele utilizar en la investigación de las TIC con variedad de estudios en este sentido: Cope y Ward, 2002; NCES, 2000; Cabero, 2000; Solmon y Wiederhorn, 2000; De Pablos y Colás, 1998; Escudero, 1989.

Con esta investigación se pretende aportar resultados reales, fruto de la investigación de campo con los alumnos durante varios cursos académicos, que ofrezcan información válida y contrastable que ayude al docente en la toma de decisiones para la mejora en los procesos educativos. Hasta 
ahora y de forma generalizada, las decisiones en este sentido se toman apoyándose en propuestas conceptuales o teóricas, sin considerar al sujeto clave objeto de dichas decisiones, el alumno. Resulta por tanto complicado facilitar un proceso de innovación en el aula a través de propuestas basadas en intuiciones de los problemas y necesidades que se dan en un centro en vez de basarse en datos obtenidos de la fuente, una vez más, el alumno.

En definitiva, este estudio pretende captar la realidad de los procesos formativos basados en el ABP y la aplicación de una detallada Acción Tutorial en la Universidad, para conocer los problemas reales que se deben tratar de solventar, los aspectos positivos que se deben reforzar y una vez captada la realidad y el mejor modo de enfocar su aplicación, por lo que este documento debe invitar a la reflexión a todos los miembros de la comunidad universitaria a incentivar e impulsar estos modelos metodológicos.

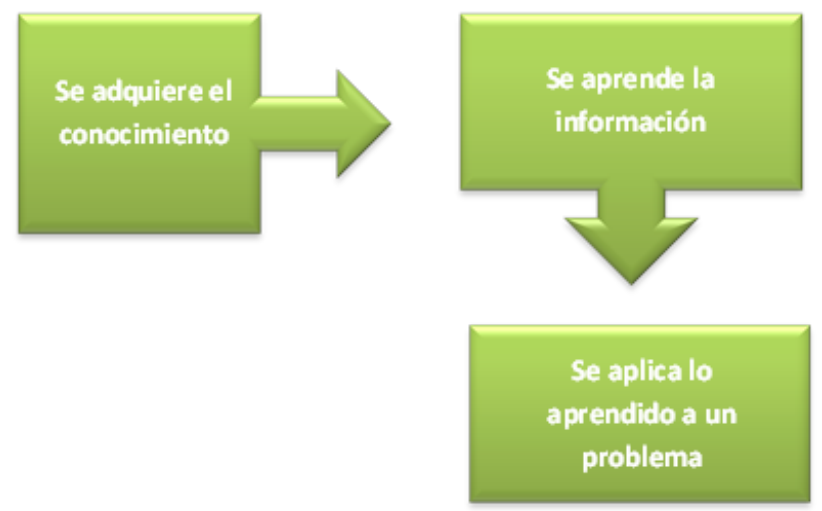

Figura 1. Proceso de Aprendizaje Tradicional. Fuente: Dirección de Investigación y Desarrollo Educativo. Vicerrectoría Académica, Instituto Tecnológico y de Estudios Superiores de Monterrey (2005).

Con los procesos de ABP se invierte el proceso de aprendizaje tradicional (Figura 1), que con los métodos convencionales primero se expone la información y posteriormente se busca su aplicación en la resolución del problema. En el caso del ABP (Figura 2) primero se presenta el problema, se identifican las necesidades de aprendizaje, se busca la información necesaria y finalmente se regresa al problema (Dirección de Investigación y Desarrollo Educativo. Vicerrectoria Académica, Instituto Tecnológico y de Estudios Superiores de Monterrey, 2005).

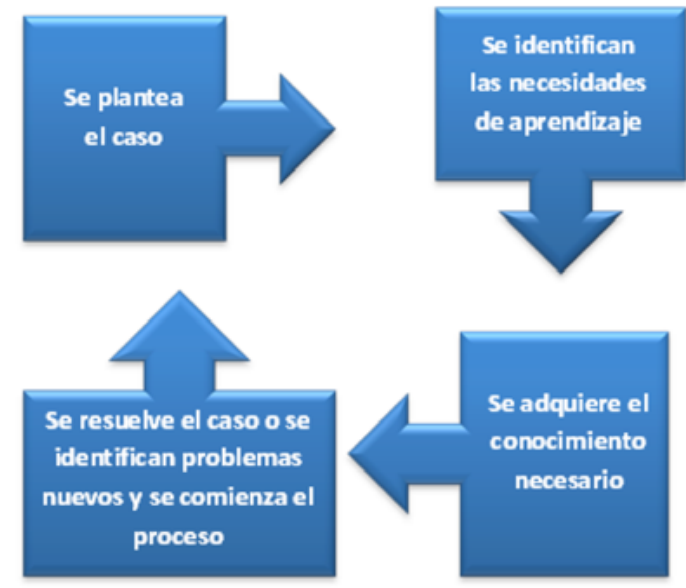


En los cursos 2009-10, 2010-11, 2011-12, 2012-13 y 2013-14 se ha desarrollado un estudio de ABP en la Unidad Docente de Caminos y Aeropuertos de la Escuela Técnica Superior de Ingeniería Civil de la Universidad Politécnica de Madrid.

Se decidió realizar el estudio sobre las asignaturas de Caminos, del Plan de Grado de Ingeniería Civil, impartida en el primer semestre, Caminos I, impartida en el primer semestre del antiguo Plan de Ingeniería Técnica de Obras Públicas y Caminos II, del segundo semestre de la misma titulación, ya que en los últimos años se estaba alcanzando una tasa de aprobados muy baja, del entorno del $15 \%$ en las tres asignaturas y una tasa de eficiencia ( $n .^{\circ}$ de aprobados $/ n{ }^{\circ}$ de presentados) del $25 \%$, también para las tres.

En una primera etapa se confeccionaron una serie de cuestionarios, con los que se pretendía conocer el perfil del alumno (procedencia, formación previa, asignaturas pendientes, expectativas respecto a las tutorías...).

Seguidamente se fueron diseñando los ejercicios, problemas y casos que deberían de resolver los alumnos a lo largo de la experiencia, a la vez que se diseñó un modelo de tutorías, tanto presenciales como a través del correo electrónico y Moodle, factible y adecuado a los alumnos, de manera que ayudara a:

- Aumentar la implicación de los alumnos durante el desarrollo de la asignatura.

- Aumentar la eficiencia de la labor del profesorado.

- Mejorar el rendimiento de los alumnos.

- Obtener una mayor satisfacción del profesor, en el desempeño de su función como docente.

En una segunda etapa se ha llevado el modelo a la práctica, que se expondrá en los siguientes puntos.

\section{Diseño}

El trabajo de investigación se ha estructurado por fases. En la Figura 3 se muestra un esquema del proceso, basado en los aspectos fundamentales, con respecto a cada una de las fases de la investigación. 


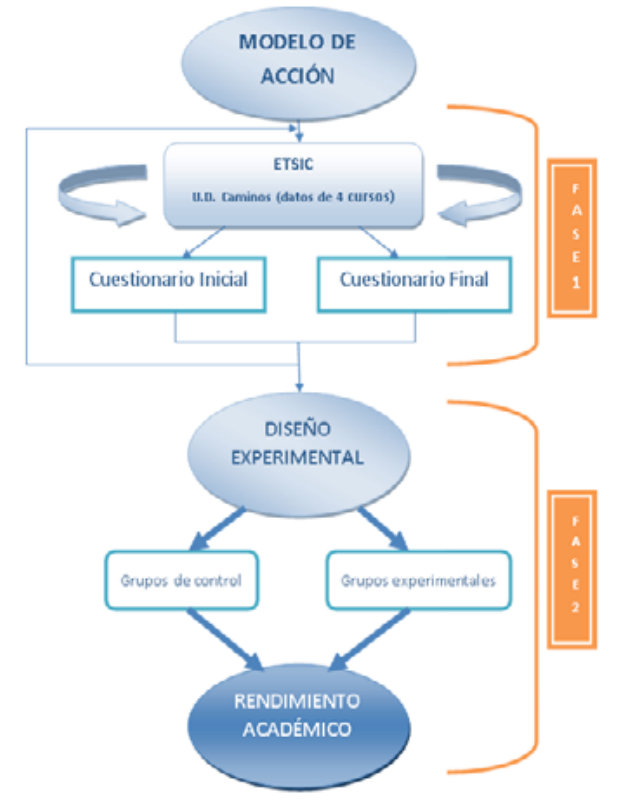

\section{Fase 1}

Figura 3. Modelo adoptado. Fuente: Elaboración propia.

En la primera fase de la investigación se realiza un Cuestionario Inicial a todos los alumnos que asisten a las clases regladas, tanto en el turno de mañana como en el de tarde, por lo que se obtienen datos de dos grupos por asignatura.

Durante el curso, además de analizar la información obtenida del cuestionario inicial, se pone en marcha una fuerte Acción Tutorial, basándose en la información obtenida del cuestionario. Asimismo, se implementa el procedimiento educativo que se van a desarrollar en la Fase 2 de la investigación (ABP).

Al finalizar el semestre los alumnos realizan un segundo cuestionario, el Cuestionario final. Se desarrolla con los alumnos que asisten a las clases regladas y en las mismas condiciones que el cuestionario inicial. Normalmente el número de alumnos encuestados resulta menor, pues en estas fechas, finales del semestre, la asistencia de los alumnos a clase es considerablemente menor debido a la carga de trabajo que acumulan.

\section{Fase 2}

En los días siguientes a la realización del cuestionario inicial se solicitan en clase alumnos voluntarios para trabajar en un grupo experimental de ABP apoyándose en las TIC.

Una vez compuestos los grupos se les informa, de forma más detallada, de cuáles son las actividades que van a realizar, el tiempo del que van a disponer para desarrollar en cada una de ellas. 


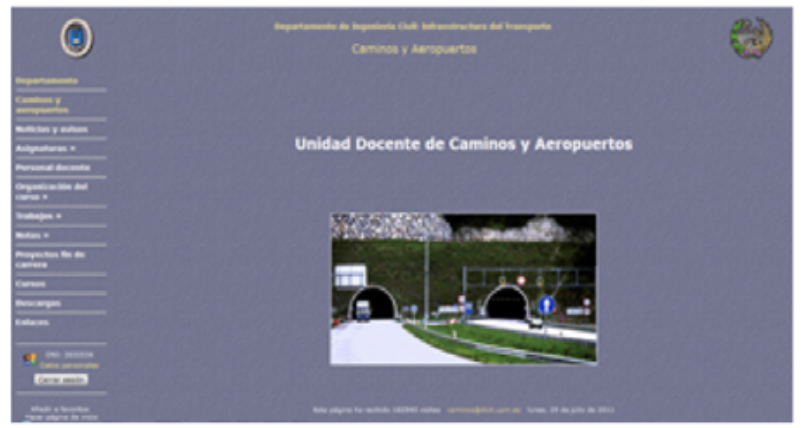

Figura 4. Web del Departamento de Ingeniería Civil: Infraestructura del Transporte.

Llegado el punto en que la información es completa se comienza con la ejecución de la Fase 2 de la investigación, que consiste en poner en marcha el ABP mediante la proposición de ejercicios/ problemas, a través de las plataformas web de que se dispone en la Universidad Politécnica de Madrid y en el propio Departamento de Ingeniería Civil: Infraestructura del Transporte (Figuras 4 y 5).

Todos los problemas se proponen, recogen y son corregidos vía e-mail. Se proponen los problemas cada lunes y el plazo máximo para entregarlos resueltos será del lunes siguiente. Durante la semana los alumnos pueden preguntar dudas sobre el problema, solicitar la bibliografía necesaria para resolverlo, solicitar confirmación del tutor de las decisiones tomadas para la resolución, siempre a través de la plataforma web, con el fin de implementar el uso de las TIC en el día a día universitario.

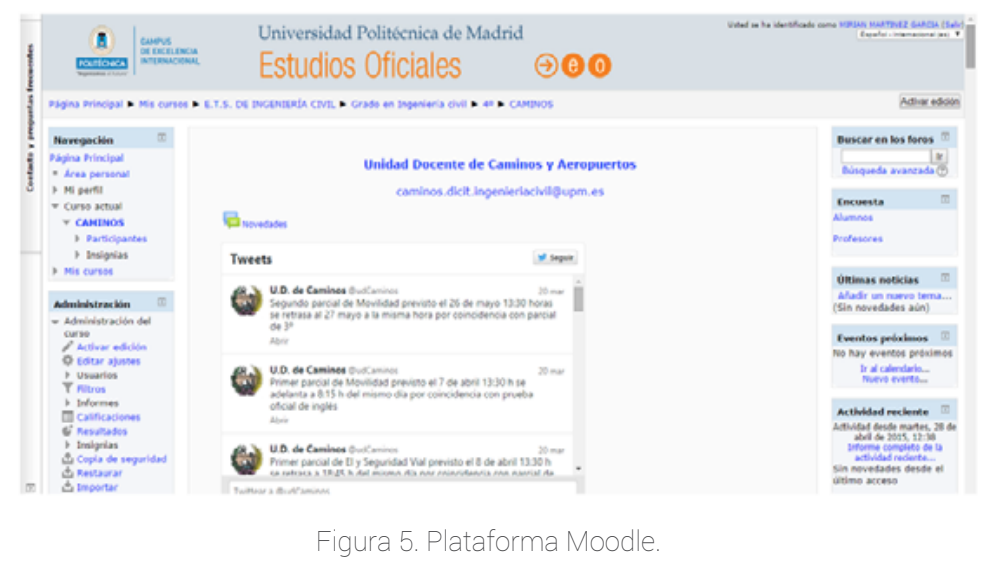

Todas las actividades han sido siempre guiadas mediante el modelo de sistema de tele-enseñanza, ya que durante todo el desarrollo del proceso los alumnos están tutorizados de forma virtual a través de todos los recursos web actuales disponibles.

Una vez finalizado el semestre los alumnos se examinarán de la asignatura a través del mismo método que el resto de los alumnos matriculados en las asignaturas en las que se ha realizado la investigación.

\section{Población y Muestra}

La elección de la muestra condiciona la validez del proceso estadístico y la interpretación de los 
resultados. La elección debe hacerse de modo aleatorio, ya que este es el que tiene mayor rigor científico. En las investigaciones de ámbito educativo es prácticamente imposible poder realizar la elección de la muestra de este modo.

La muestra elegida para la Fase 1 de la investigación tiene las características de representatividad de la población universitaria, de carácter probabilístico y no aleatoria, ya que deben ser alumnos que estén matriculados en la UPM, en la Escuela Técnica Superior de Ingeniería Civil y en concreto estar cursando, al menos, alguna de las siguientes asignaturas:

- Caminos I, asignatura impartida en el primer semestre. Titulación de Ingeniería Técnica de Obras Públicas (ITOP).

- Caminos II, asignatura impartida en el segundo semestre. Titulación de Ingeniería Técnica de Obras Públicas (ITOP).

- Caminos, asignatura impartida en el primer semestre. Titulación de Ingeniería Civil (Graduado IC).

La muestra se ha clasificado por asignaturas, quedando distribuida tal y como se puede observar en la Tabla 1 que a continuación de incluye.

\begin{tabular}{|c|c|c|}
\hline & Cuestionario Inicial & Cuestionario Final \\
\hline Caminos I & 250 & 235 \\
\hline Caminos II & 223 & 236 \\
\hline Caminos & 82 & 82 \\
\hline Totales & 633 & 608 \\
\hline
\end{tabular}

Tabla 1. Distribución de la muestra por asignaturas en la Fase 1.

La muestra de población que se selecciona para la Fase 2 se compone de alumnos que están matriculados en las asignaturas citadas en el párrafo anterior y que se han presentado de forma voluntaria para practicar los métodos de aprendizaje que se desarrollan en esta fase de la investigación, luego tampoco es una muestra seleccionada de forma aleatoria.

\begin{tabular}{|c|c|}
\hline Caminos I & 96 \\
\hline Caminos II & 78 \\
\hline Caminos & 9 \\
\hline Total & $\mathbf{1 8 3}$ \\
\hline
\end{tabular}

Tabla 2. Distribución de la muestra Fase 2

La investigación se lleva a cabo durante los cursos 2009-10, 2010-11, 2011-12 y 2013-14. Durante el curso 2012-13 no se realiza la experiencia ya que no hubo docencia debido a la implantación del nuevo plan de estudios. 


\section{Instrumentos}

En este trabajo se desarrollan investigaciones con características de naturaleza y metodología diferenciadas, pues se trata de posibilitar una complementariedad de métodos e instrumentos que permitan obtener información desde diferentes perspectivas. Para ello, en este estudio se contemplan:

\section{- Dos cuestionarios}

Cuestionario Inicial: Este cuestionario se aplica al comienzo del semestre, de ahí su nombre. Es un cuestionario que recoge las expectativas que tienen los nuevos alumnos de las asignaturas sobre la Acción Tutorial y el uso de las TIC en el proceso de enseñanza-aprendizaje, así como las opiniones que tienen los alumnos repetidores, desde su experiencia, sobre las dos acciones.

La primera parte del cuestionario recoge información descriptiva de la muestra, información como,

- Edad, sexo, Cursos en los que está matriculado, procedencia del alumnos (Bachillerato, Otra carrera universitaria, COU, FP...), si realiza algún tipo de trabajo remunerado, número de veces matriculados en la asignatura...

La segunda parte del cuestionario inicial está diseñada con preguntas más específicas sobre las expectativas que tienen sobre la Acción Tutorial y el uso de las TIC en el proceso de enseñanza-aprendizaje. El diseño de esta segunda parte se realiza con una estructura mixta, de preguntas abiertas y cerradas, que responden a los siguientes objetivos:

- Valorar si la Acción Tutorial favorece la relación profesor-alumno, valorar la información recibida hasta ahora por internet, valorar las expectativas y los conocimientos de los alumnos respecto a la Acción Tutorial y el uso de las TIC en el proceso de enseñanzaaprendizaje.

- Conocer su disposición para participar en asignaturas a través de la red, así como su disponibilidad de trabajo (infraestructura, recursos...).

- Valorar la opinión que sobre la Acción Tutorial y el uso de las TIC en el proceso de enseñanza-aprendizaje, valorar si la utilización de la Acción Tutorial y de las TIC a la hora de llevar la asignatura al día, mejorar el método de estudio, entender mejor la asignatura, mejorar el rendimiento académico, facilita la interacción profesor-alumno 
y ayuda al trabajo en grupo.

Cuestionario Final: muestra hasta qué punto las expectativas del alumnos se han visto satisfechas o no después de haber experimentado el modelo de Acción Tutorial y el uso de las TIC en el proceso de enseñanza-aprendizaje.

Los cuestionarios se aplican al finalizar el semestre y siempre antes de realizar la evaluación para que estos no se vean afectados por los resultados de la misma.

La estructura del cuestionario es similar a la del Cuestionario Inicial, una primera parte con una serie de preguntas de carácter general, para identificar la muestra, y una segunda parte del cuestionario que está diseñado con preguntas más específicas sobre la opinión que tienen de la Acción Tutorial y el uso de las TIC en el proceso de enseñanza-aprendizaje una vez finalizado el curso. El diseño de esta segunda parte, al igual que en el cuestionario inicial, se realiza con una estructura mixta, de preguntas abiertas y cerradas, que responden a los siguientes objetivos:

- Valorar la opinión sobre las tutorías, en cuanto a la frecuencia con que las han utilizado, el uso que han hecho de ellas, valorar la información que han recibido durante el curso por internet, valorar las expectativas y los conocimientos de los alumnos respecto a la Acción Tutorial y el uso de las TIC en el proceso de enseñanza-aprendizaje, valorar la opinión sobre la Acción Tutorial y el uso de las TIC en el proceso de enseñanzaaprendizaje, valorar las actitudes que han hecho que el alumno acuda a las tutorías, valorar si la tele-enseñanza favorece la relación profesor-alumno, valorar si la utilización de la Acción Tutorial y de las TIC les ha hecho llevar la asignatura al día, integrarse en la escuela, mejorar el método de estudio, entender mejor la asignatura, mejorar el rendimiento académico, facilita la interacción profesor-alumno y ayuda al trabajo en grupo.

- Ejercicios de realización individual y semanal, en el ABP los roles del alumno y del profesor cambian respecto a los métodos tradicionales de enseñanza, en este caso los roles son los que se muestran a continuación en la Tabla 3.

\begin{tabular}{|c|c|}
\hline \multicolumn{2}{|c|}{ ROLES } \\
\hline ALUMNO & PROFESOR \\
\hline Asumir su responsabilidad ante el aprendizaje & $\begin{array}{c}\text { Dar un papel protagonista al alumno en la } \\
\text { construcción de su aprendizaje }\end{array}$ \\
\hline $\begin{array}{c}\text { Ser autónomo en el aprendizaje (buscar } \\
\text { información, contrastarla, comprenderla, aplicarla, } \\
\text { etc.) }\end{array}$ & $\begin{array}{c}\text { Ser consciente de los logros que consiguen sus } \\
\text { alumnos }\end{array}$ \\
\hline
\end{tabular}




\begin{tabular}{|c|c|}
\hline Saber pedir ayuda & $\begin{array}{c}\text { Ser un facilitador del aprendizaje, acudiendo a } \\
\text { los alumnos cuando le necesitan y que les ofrece } \\
\text { información cuando la necesitan }\end{array}$ \\
\hline Compartir información y aprender de los demás & $\begin{array}{c}\text { El papel principal es ofrecer a los alumnos diversas } \\
\text { oportunidades de aprendizaje }\end{array}$ \\
\hline Pedir orientación cuando lo necesite & $\begin{array}{c}\text { Ayudar a sus alumnos a que piensen críticamente } \\
\text { orientando sus reflexiones y formulando } \\
\text { cuestiones importantes }\end{array}$ \\
\hline $\begin{array}{c}\text { Disponer de las estrategias necesarias para } \\
\text { planificar, controlar y evaluar los pasos que lleva a } \\
\text { cabo en su aprendizaje }\end{array}$ & Realizar sesiones de tutoría con los alumnos \\
\hline
\end{tabular}

Tabla 3. Rol del Profesorado y papel de los alumnos. Fuente: Servicio de Innovación educativa de la UPM.

La implementación en las aulas la aplicación del ABP, apoyándose de las TIC como forma de comunicación y obtención de información y Acción Tutorial se realiza vía e-mail.

Se proponen los problemas cada lunes y el plazo máximo para entregarlos resueltos será del lunes siguiente. Durante la semana los alumnos pueden preguntar dudas sobre el problema, solicitar la bibliografía necesaria para resolverlo, solicitar confirmación del tutor de las decisiones tomadas para la resolución... siempre a través de la plataforma web, con el fin de implementar el uso de las TIC en el día a día universitario.

La propuesta de ejercicios se realiza en semanas alternas, de forma que los alumnos elaboran seis ejercicios teórico/prácticos con este proceso metodológico durante el semestre.

Todas estas actividades están siempre guiadas mediante el modelo de sistema de teleenseñanza, ya que durante todo el proceso lo alumnos están tutorizados de forma virtual, puesto que para este proceso de teleenzañanza se integran todos los recursos web actuales disponibles.

- Actas de evaluación de las asignaturas, en la fase final de la investigación la valoración de la experiencia se realiza a través de la comparativa entre los resultados obtenidos en los exámenes ordinarios por los alumnos que has desarrollado la asignatura dentro del grupo de investigación y los alumnos que la han desarrollado con los métodos convencionales. Este análisis se realiza utilizando como instrumento las actas oficiales de evaluación de los alumnos en cada una de las convocatorias anuales.

\section{Análisis de datos}

A continuación se presentan los principales resultados obtenidos en el estudio, ya sean sobre calificaciones o sobre nivel de satisfacción. 
Análisis comparativo de los resultados obtenidos mediante el aprendizaje basado en problemas junto con una acción tutorial apoyada en un modelo de teleensañanza y los metodos convencionales de enseñanza

El principal medio del que se dispone para medir los resultados obtenidos son las evaluaciones, las calificaciones casi nunca ofrecen valores relativos a los aspectos anteriores, aunque sí marcan el grado de efectividad del método y el nivel de satisfacción de los alumnos.

Los datos recogidos en las Figuras 6, 7 y 8 muestran la tasa de eficiencia en función del método de aprendizaje utilizado, cabe destacar que, independientemente de la convocatoria que se estudie, la dispersión es menor si el método utilizado es el de ABP que por métodos convencionales en cada una de las asignaturas analizadas en el estudio.

En la asignatura de Caminos I se puede observan que la tasa de eficiencia para el conjunto de todos los alumnos que están matriculados no supera en ningún curso el 50\%. Es importante destacar que el número de alumnos no presentados, en el curso 2013-14, ha tenido un valor del $47 \%$.

En cuanto a los resultados obtenidos por los alumnos que han participado en la investigación, la tasa de eficiencia tiene unos valores medios del 68\%. El curso 2010-11, primer año de la fase de experimentación, se obtienen valores del 38\%. En el resto de los cursos el valor de la tasa de eficiencia ha ascendido hasta llegar a valores del 92\% en el curso 2013-14.
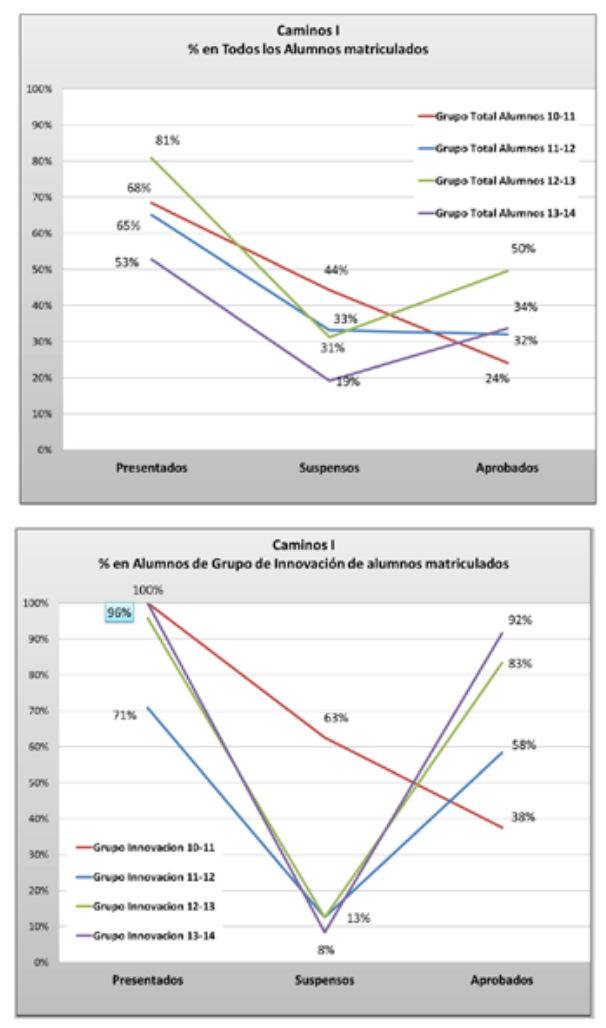

Figura 6. Tasa de eficiencia interanual. Caminos I. 
Se observa que el comportamiento del conjunto de los alumnos matriculados de la asignatura de Caminos II, en cuanto a tasa de eficiencia se refiere, tiene unos valores medios del 37\%, no superando el $50 \%$ en ningún caso. Mientras que para el grupo de los alumnos que han participado en la investigación la tasa de eficiencia ha adquirido unos valores medios del 62\%.

El curso 2010-11, primer año de la fase de experimentación, se han obtenido valores del 48\%. El resto de los cursos el valor de la tasa de eficiencia ha ascendido hasta llegar a valores del $81 \%$ en el curso 2013-14.
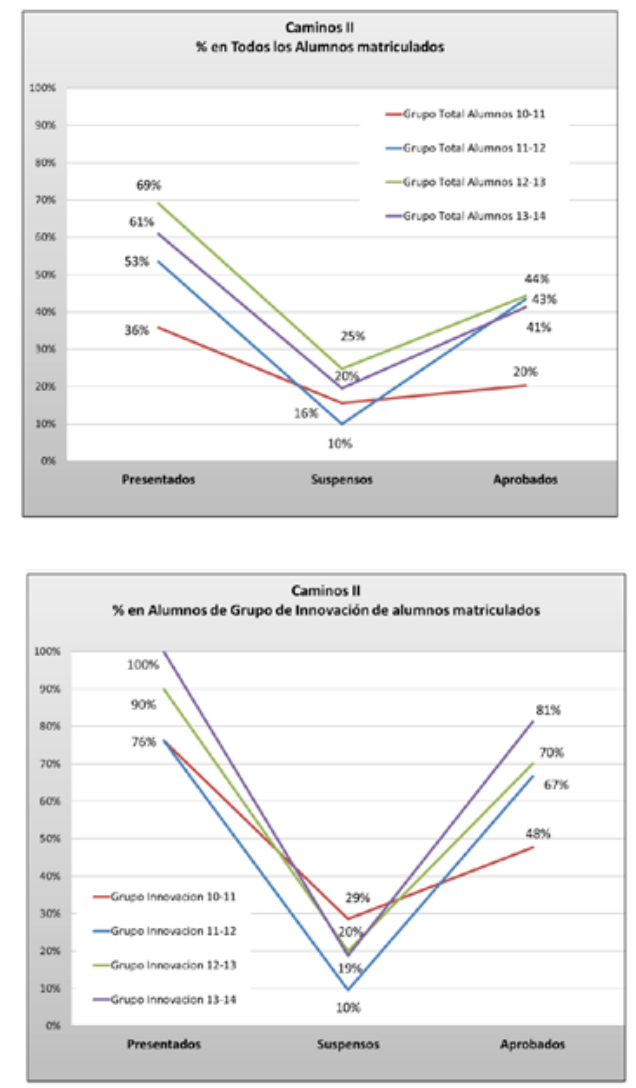

Figura 7. Tasa de eficiencia interanual. Caminos II.

Se observa que el comportamiento de la tasa de eficiencia del conjunto de los alumnos matriculados de la asignatura de Caminos tiene un valor del $54 \%$ frente al $67 \%$ que posee la tasa de los alumnos que han colaborado en la investigación.

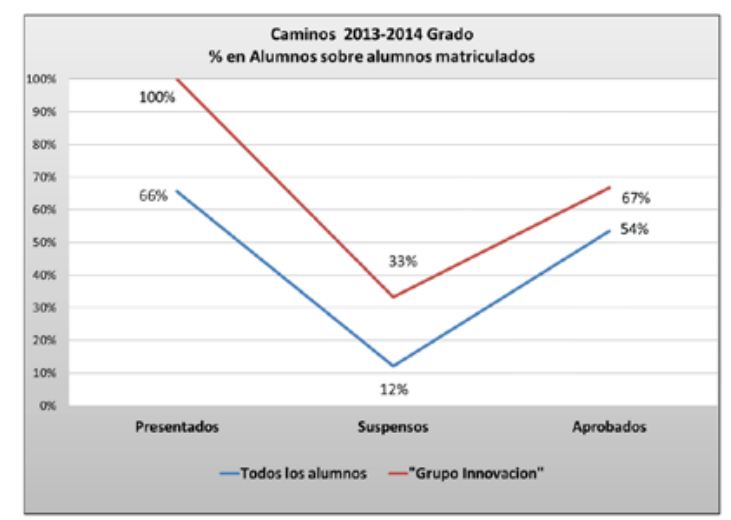

Figura 8. Tasa de eficiencia interanual. Caminos. 
En esta asignatura el porcentaje de no presentados del grupo de alumnos que asiste a la docencia de forma ordinaria es del $44 \%$.

Según se fue repitiendo la experiencia año tras año los resultados fueron mejorando de una forma exponencial, Ilegando a tener unas tasas de eficiencia del 90\% en el curso 2013-14, tal y como muestra la Figura 6 en la asignatura de Caminos I.

Esta gran mejora de los resultados se debe a una mayor implicación de los alumnos, cada año más familiarizados con el método y con la forma de afrontar las asignaturas, olvidándose de los métodos tradicionales, haciendo que los alumnos desarrollen más sus capacidades de identificar y resolver problemas (Inda Caro \& Álvarez González, 2008).

Y de forma simultánea, una mayor implicación de los profesores, que han ido centrando su trabajo cada vez más a enfocar los métodos de enseñanza a la adquisición de las competencias, ha hecho que los alumnos obtengan además de mayores conocimientos mejores resultados.

\section{Análisis del grado de satisfacción de los alumnos que han utilizado el aprendizaje basado en problemas junto con una acción tutorial apoyada en un modelo de teleenseñanza}

Al finalizar la experiencia, los alumnos que participaron en el proceso realizaron un cuestionario final, antes de la realización del examen, con el que se pretendía conocer si el modelo de teleenseñanza:

- Mejora el rendimiento académico

- Se integran mejor en la escuela

- Ayuda a llevar la asignatura al día

- Entienden mejor la asignatura

- Mejora el método de estudio

- Mejora el pensamiento crítico

- Mejora la creatividad, la capacidad de identificar y resolver problemas

- Mejora las habilidades comunicativas

- Facilita la interacción profesor-alumno

Tras analizarlos, se puede afirmar que la mayor parte de los alumnos se han mostrado favorables al método implantado. Con él han conseguido adquirir competencias tales como el pensamiento crítico, la creatividad, capacidad de identificar y resolver problemas, habilidades comunicativas, desarrollar el 
aprendizaje auto-dirigido, la interacción profesor-alumno y el trabajo en grupo.

De modo que, midiendo en una escala en la que 1 es nada de acuerdo y 5 totalmente de acuerdo, los datos obtenidos de los cuestionarios se pueden conocer de forma global en las Tablas 4, 5, 6, 7, 8 y 9.

En las tablas 4 y 5, se comprueba cuál ha sido la variación de opinión de los alumnos de la asignatura de Caminos I a lo largo del curso respecto a la ayuda que la información que han recibido de internet les podría proporcionar de modo que tanto al principio de curso como al final de mismo es Entender mejor la asignatura es la opción elegida por los alumnos encuestados en periodos de tiempo.

En cuanto al resto de respuestas se observa que la opción menos valorada por los encuestados del cuestionario inicial es la de Ayuda al trabajo en grupo y en el cuestionario final, con igualdad de valoración son la Ayuda al trabajo en grupo, Integrarte en la escuela y Facilitar la interacción profesoralumno.

\begin{tabular}{|c|c|c|c|c|c|}
\hline PUNTUACIÓN (\%) & $\mathbf{1}$ & $\mathbf{2}$ & $\mathbf{3}$ & $\mathbf{4}$ & $\mathbf{5}$ \\
\hline Llevar la asignatura al día & 24,8 & 14,4 & 13,2 & 8 & 5,6 \\
\hline Mejorar tu método de estudio & 27,2 & 12 & 17,2 & 8,4 & 2,8 \\
\hline $\begin{array}{c}\text { Entender mejor la asignatura } \\
\text { Mejorar tu rendimiento } \\
\text { académico }\end{array}$ & 14,4 & 13,2 & 19,6 & 10,4 & 6 \\
\hline $\begin{array}{c}\text { Facilita la interacción profesor- } \\
\text { alumno }\end{array}$ & 26,8 & 18,8 & 13,2 & 4,4 & 1,6 \\
\hline \begin{tabular}{c} 
Ayuda al trabajo en grupo \\
\hline
\end{tabular} & 42,8 & 14,8 & 7,6 & 4,4 & 0,4 \\
\hline
\end{tabular}

Tabla 4. Avuda que proporciona la información obtenida en la Acción Tutorial (\%). Comparativa Cl. Caminos I.

\begin{tabular}{|c|c|c|c|c|c|}
\hline PUNTUACIÓN (\%) & $\mathbf{1}$ & $\mathbf{2}$ & $\mathbf{3}$ & $\mathbf{4}$ & $\mathbf{5}$ \\
\hline $\begin{array}{c}\text { Mejorar tu rendimiento } \\
\text { académico }\end{array}$ & 17,02 & 27,23 & 20,43 & 14,89 & 4,26 \\
\hline Integrarte en la escuela & 23,40 & 18,30 & 14,47 & 3,40 & 1,28 \\
\hline Llevar la asignatura al día & 19,57 & 23,40 & 25,11 & 10,21 & 4,26 \\
\hline Entender mejor la asignatura & 15,32 & 24,26 & 29,36 & 18,30 & 6,81 \\
\hline Mejorar tu método de estudio & 22,13 & 20,85 & 19,15 & 12,77 & 5,11 \\
\hline Facilita la interacción profesor- & 21,28 & 20,00 & 18,30 & 14,47 & 1,28 \\
\hline alumno & 26,81 & 18,72 & 7,66 & 5,96 & 1,28 \\
\hline Ayuda al trabajo en grupo
\end{tabular}


En cuanto a la ayuda que proporciona la información obtenida en las tutorías para los alumnos de la asignatura de Caminos II se puede comprobar en las Tablas 6 y 7 cuál ha sido la variación de opinión a lo largo del curso, de modo que al principio de curso la ayuda más valorada es Entender mejor la asignatura y al final de curso es Mejorar tu rendimiento académico.

\begin{tabular}{|c|c|c|c|c|c|}
\hline PUNTUACIÓN (\%) & $\mathbf{1}$ & $\mathbf{2}$ & $\mathbf{3}$ & $\mathbf{4}$ & $\mathbf{5}$ \\
\hline Llevar la asignatura al día & 24,8 & 14,4 & 13,2 & 8 & 5,6 \\
\hline Mejorar tu método de estudio & 27,2 & 12 & 17,2 & 8,4 & 2,8 \\
\hline Entender mejor la asignatura & 14,4 & 13,2 & 19,6 & 10,4 & 6 \\
\hline $\begin{array}{c}\text { Mejorar tu rendimiento } \\
\text { académico }\end{array}$ & 18,4 & 12 & 19,6 & 12 & 1,6 \\
\hline $\begin{array}{c}\text { Facilita la interacción profesor- } \\
\text { alumno }\end{array}$ & 26,8 & 18,8 & 13,2 & 4,4 & 4,8 \\
\hline Ayuda al trabajo en grupo & 42,8 & 14,8 & 7,6 & 4,4 & 0,4 \\
\hline
\end{tabular}

Tabla 6. Ayuda que proporciona la información obtenida en las Tutorías (\%). Comparativa Cl. Caminos II.

\begin{tabular}{|c|c|c|c|c|c|}
\hline PUNTUACIÓN (\%) & $\mathbf{1}$ & $\mathbf{2}$ & $\mathbf{3}$ & $\mathbf{4}$ & $\mathbf{5}$ \\
\hline Mejorar tu rendimiento académico & 15,68 & 29,24 & 24,58 & 5,51 & 4,24 \\
\hline Integrarte en la escuela & 18,64 & 23,31 & 22,88 & 2,97 & 0,42 \\
\hline Llevar la asignatura al día & 30,51 & 21,19 & 15,25 & 8,05 & 3,39 \\
\hline Entender mejor la asignatura & 19,92 & 31,78 & 18,64 & 12,71 & 2,54 \\
\hline Mejorar tu método de estudio & 30,08 & 22,46 & 16,10 & 8,90 & 0,85 \\
\hline Facilita la interacción profesor- & 18,22 & 30,51 & 13,14 & 13,14 & 0,85 \\
\hline alumno & 28,81 & 24,58 & 5,08 & 9,75 & 0,85 \\
\hline
\end{tabular}

Tabla 7. Ayuda que proporciona la información obtenida en las Tutorías (\%). Comparativa CF. Caminos II.

Los alumnos de la asignatura de Caminos han valorado la ayuda proporcionada por la tutorías tal y como se muestra en las Tablas 8 y 9, en las que se puede comprobar cuál ha sido la variación de opinión a lo largo del curso, de modo que al principio de curso la ayuda más valorada es Entender mejor la asignatura y al final de curso es Mejorar tu rendimiento académico.

\begin{tabular}{|c|c|c|c|c|c|}
\hline PUNTUACIÓN (\%) & $\mathbf{1}$ & $\mathbf{2}$ & $\mathbf{3}$ & $\mathbf{4}$ & $\mathbf{5}$ \\
\hline Llevar la asignatura al día & 23,81 & 23,81 & 7,14 & 32,14 & 13,10 \\
\hline Mejorar tu método de estudio & 36,90 & 28,57 & 9,52 & 19,05 & 5,95 \\
\hline Entender mejor la asignatura & 21,43 & 11,90 & 27,38 & 10,71 & 28,57 \\
\hline
\end{tabular}




\begin{tabular}{|c|c|c|c|c|c|}
\hline $\begin{array}{c}\text { Mejorar tu rendimiento } \\
\text { académico }\end{array}$ & 28,57 & 19,05 & 29,76 & 16,67 & 5,95 \\
\hline $\begin{array}{c}\text { Facilita la interacción profesor- } \\
\text { alumno }\end{array}$ & 27,38 & 34,52 & 16,67 & 13,10 & 8,33 \\
\hline Ayuda al trabajo en grupo & 55,95 & 14,48 & 10,90 & 8,33 & 8,33 \\
\hline
\end{tabular}

Tabla 8. Ayuda que proporciona la información obtenida en las Tutorías (\%). Comparativa Cl. Caminos.

\begin{tabular}{|c|c|c|c|c|c|}
\hline PUNTUACIÓN (\%) & $\mathbf{1}$ & $\mathbf{2}$ & $\mathbf{3}$ & $\mathbf{4}$ & $\mathbf{5}$ \\
\hline Mejorar tu rendimiento académico & 17,07 & 26,83 & 21,95 & 14,63 & 4,88 \\
\hline Integrarte en la escuela & 24,39 & 17,07 & 14,63 & 3,66 & 1,22 \\
\hline Llevar la asignatura al día & 17,07 & 23,17 & 28,05 & 10,98 & 6,10 \\
\hline Entender mejor la asignatura & 13,41 & 21,95 & 30,49 & 20,73 & 8,54 \\
\hline Mejorar tu método de estudio & 20,73 & 21,95 & 20,73 & 12,20 & 6,10 \\
\hline $\begin{array}{c}\text { Facilita la interacción profesor- } \\
\text { alumno }\end{array}$ & 23,17 & 21,95 & 15,85 & 12,20 & 1,22 \\
\hline Ayuda al trabajo en grupo & 28,05 & 19,51 & 8,54 & 4,88 & 1,22 \\
\hline
\end{tabular}

Tabla 9. Ayuda que proporciona la información obtenida en las Tutorías (\%). Comparativa CF. Caminos.

Es importante considerar que, en los primeros años, algunos alumnos sugieren que tanto la información recibida como el trabajo realizado no es suficiente para superar la asignatura, aun así todos ellos estarían dispuestos a repetir la experiencia en esta u otra asignatura.

\section{Conclusiones}

El objetivo fundamental del estudio ha sido tratar de determinar los efectos sobre las calificaciones de los alumnos y su satisfacción por el Aprendizaje Basado en Problemas, mediante un Modelo de Sistema de Teleenseñanza como acción tutorial frente a los métodos de aprendizaje convencionales.

De los resultados y experiencias presentados anteriormente, se puede concluir que una práctica pedagógica como la estudiada es una alternativa que produce unos mejores resultados o al menos más acordes con lo que se plantea que debe ser la formación universitaria.

Asimismo, aunque en los primeros años no se observaban mejorías significativas en las calificaciones, en la adquisición de conocimiento sí la había, el solo hecho de mejorar significativamente el entorno de aprendizaje del futuro ingeniero, generando una mayor satisfacción de profesores y alumnos, puede considerarse una meta en sí misma. 
Mediante la utilización de este método se ha logrado incrementar el tiempo de dedicación e implicación con las asignaturas. Hecho que ha dado lugar a mejores calificaciones y menor abandono de la asignatura.

La realización de la resolución de los casos ha supuesto para el alumno un considerable número de horas de trabajo fuera del aula. En el seguimiento realizado, estaba alrededor del 55 por ciento del tiempo, que se ha dedicado fundamentalmente a las tareas de búsqueda de información, análisis y diseño de soluciones.

La incorporación de las nuevas tecnologías ha facilitado el trabajo colaborativo de los alumnos y las tareas de seguimiento y evaluación del docente.

Se han detectado algunas carencias en la preparación de los alumnos para enfrentarse al análisis, valoración y selección de alternativas que les permita realizar una toma de decisiones fundamentada. Esto se debe fundamentalmente a que los alumnos están habituados a resolver problemas cerrados, con una solución única y no a tomar decisiones, algo fundamental en su futuro trabajo, en este caso como ingenieros.

Desde el punto de vista negativo de la experiencia, se puede afirmar que en los alumnos que comienzan a trabajar en el segundo semestre su implicación con el proyecto es mucho menor que los que comienzan desde principio de curso.

Por último, cabe destacar el hecho de que, independientemente de los resultados obtenidos por los alumnos en las evaluaciones, el grado de satisfacción del alumno con la formación recibida y lo aprendido es muy elevado.

\section{Referencias}

Area, M. (2005). Tecnologías de la información y la comunicaciión en el sistema escolar. Una revisión de las líneas de investigación. Relieve, 17(1), 3-25.

Cabero, J. (Ed.) (2000). Uso de los medios audiovisuales, informáticos y las nuevas tecnologías en los centros andaluces. Sevilla, España: Konos.

Cope, C., \& Ward, P. (2002). Integrating learning technology into classrooms: The importance of teachers' perceptions. Educational Technology \& Society, 5(1).

Cuban, L. (2001). Oversold and Underused: Computers in the classroom. London, UK: Harvard University.

De Pablos, J., \& Colás, P. (Eds.). (1998). La implantación de las nuevas tecnologías de la información 
y comunicación en el sistema educativo andaluz: un estudio evaluativo. Sevilla, España: Grupo de investigación evaluación y tecnología educativa, Universidad de Sevilla.

Dirección de Investigación y Desarrollo Educativo - Vicerrectoría Académica. (2005). El aprendizaje basado en problemas como técnica didáctica. Monterrey, México

Escudero, J. M. (Ed.) (1989). Evaluación del proyecto Atenea. Informe de Progreso. Programa de Nuevas Tecnologías de la Información y Comunicación. Madrid, España: MEC.

Inda Caro, M., Álvarez González, S., \& Álvarez Rubio, R. (2008). Métodos de evaluación en la enseñanza superior. Revista de Investigación Educativa, 26(2), 539-552.

Salinas, J., Darder, A., \& De Benito, B. (2015). Las TIC en la enseñanza superior: e-learning, b-learning y m-learning. In J. Cabero \& J. Barroso (Eds.), Nuevos retos en tecnología educativa (pp. 153-174). Madrid, España: Síntesis.

Servicio de Innovación Educativa de la Universidad Politécnica de Madrid. (2008). Aprendizaje Basado en Problemas. Madrid, España: http://innovacioneducativa.upm.es/guias/Aprendizaje_basado_en_ problemas.pdf

Solmon, L., \& Wiederhorn, J. (2000). Progress of Technology in the Scool: 1999. Report on 27 states. Milken Exchange on Education and Technology. Santa Monica, CA, USA: Milken Family Foundation. 